\title{
Brasil y su política nacional de salud bucal
}

\section{National oral health policy in Brazil}

\section{Resumen}

El programa Brasil Sonriente (BS) fue creado por el gobierno federal de Brasil, como parte de su Sistema Único de Salud, en el año 2004. Dicho programa provee decenas de miles de equipos de salud bucal en la atención primaria básica, establece atenciones de mediana complejidad y fomenta las políticas municipales de fluorización del suministro de agua y rehabilitación bucal protésica, por medio de los laboratorios regionales de prótesis dental, entre otras acciones. La Política Nacional de Salud Bucal (PNSB) y su programa BS son reconocidos como responsables del aumento en el acceso a los servicios de salud pública. Aproximadamente 15 años después de la implementación de la PNSB, pueden ser observados avances importantes: aumento exponencial en la cobertura de los equipos de salud bucal en la atención básica y un enfoque del cuidado integral de la salud. Sin embargo, los puntos positivos no pueden ocultar los obstáculos que aún existen. La municipalización del sistema de salud se llevó a cabo bajo el amparo de la redemocratización y la premisa del empoderamiento de los actores locales en la discusión de un modelo de salud más conveniente para ofrecer servicios a los usuarios. Sin embargo, la forma en que el Estado brasilero se organiza y financia distorsionó la estructura de las redes integrales de atención en salud.

Palabras clave: Administración en salud pública; Salud bucal; Sistema único de salud (fuente: DeCS BIREME).

\section{Abstract \\ The Smiling Brazil (BS) program was created by the federal government of Brazil, as part of its Unified Health System, in 2004. This program provides tens of thousands of oral health teams in basic primary care, establishes care for medium complexity and promotes municipal policies for fluoridation of the water supply and prosthetic oral rehabilitation, through regional dental prosthesis laboratories, among other actions. The National Oral}

\section{Nota Científica}

Sylvio da Costa Junior ${ }^{1, a}$, Ximena Concha Melgar ${ }^{1, b}$, Fernando Neves Hugo ${ }^{1, a}$

1 Universidade Federal do Rio Grande do Sul (UFRGS), Porto Alegre, Brasil.

a Doctor en Odontología, Àrea de Salud Bucal Colectiva

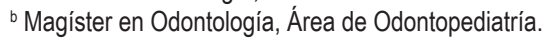

\section{Correspondencia:}

Fernando Neves Hugo: fernandoneveshugo@gmail.com Rua Ramiro Barcelos 2492. Porto Alegre, RS, Brasil. CEP 90035-003

ORCID: 0000-0003-2222-7719

\section{Coautores:}

Ximena Concha Melgar: xconchamelgar@gmail.com ORCID: 0000-0002-5426-4728

Sylvio da Costa Junior: sylviocosta13@gmail.com ORCID: 0000-0003-0218-5515

\section{Editora:}

Rosse Mary Falcón-Antenucci

Universidad Inca Garcilaso de la Vega, Perú.

Conflicto de intereses: los autores declaran no tener conflictos de interés.

Fuente de financiamiento: ninguna.

Recibido: $30 / 08 / 20$

Aceptado: $14 / 09 / 20$

Publicado: $16 / 11 / 20$ 
Health Policy (PNSB) and its BS program are recognized as responsible for increasing access to public health services. Approximately 15 years after the implementation of the PNSB, important advances can be observed: exponential increase in the coverage of oral health teams in basic care and a comprehensive health care approach. However, the accomplishments cannot hide the obstacles that still exist. The municipalization of the health system was carried out under the protection of democratization and the premise of empowerment of local officers in the discussion of a more convenient health model to offer services to users. But the way in which the Brazilian State organized and financed itself distorted the structure of the health care networks.

Keywords: Public health administration; Oral health; Unified health system (source: MeSH NLM).

\section{Introducción}

La construcción de los sistemas de atención y cuidado de la salud es una respuesta política frente a las demandas y preferencias de la sociedad. En ese sentido, estos deben ser articulados por las necesidades de salud de la población, que se expresan, en gran parte, en situaciones demográficas, sociales y epidemiológicas particulares ${ }^{1}$. Con la instauración de la ley No 4.682, de autoría del diputado federal Eloy Chaves, durante el gobierno del Presidente de la República Arthur Bernardes, el 24 de enero de 1923 se instituyó la primera política de seguridad social en el Brasil ${ }^{2}$.

Este modelo de seguridad social se basó en el mismo pilar que el modelo del Welfare State, que proporcionó beneficios a cambio de contribuciones de los trabajadores asalariados que formaban la naciente industria nacional ${ }^{3}$. Inicialmente, solo los trabajadores vinculados a las actividades relacionadas con los ferrocarriles, podrían disfrutar de este derecho. La ley abrió la posibilidad que los trabajadores formales, empleados en esta actividad específica, creen sus fondos de jubilación y pensión (CAPs), con el objetivo de que accedan a servicios de atención médica y servicios de pago de jubilación y pensiones, lo cual funcionaba bajo el régimen de capitalización.

En el modelo del Welfare State adoptado inicialmente en Brasil (conservador o corporativo), el gobierno intervendría sólo para resolver conflictos entre las partes, ya que la administración sería organizada por trabajadores y empleados. Con un vertiginoso crecimiento de recursos a disposición de las CAPs en 1930 se instituyeron los Institutos de Jubilaciones y Pensiones (IAPs), con la diferencia significativa de una mayor administración del gobierno sobre esos recursos y extensión a los demás trabajadores pertenecientes al mercado laboral formal. Con los IAPs, la odontología se incluyó en la lista de servicios ofrecidos a los asegurados. Pero ésta tenía un fuerte carácter mutilador y estaba centrada en la enfermedad ${ }^{4,5}$.

Paralelamente a ese modelo de asistencia, la salud bucal no tuvo un estándar nacionalizado de asistencia hasta $1947^{4,6,7}$. Varios estados contaron con programas específicos de servicios de higiene bucal para algunos grupos poblacionales, como reclusos o inspecciones médico-escolares, en ausencia de planificación, promoción y tratamiento continuo, además de ser focalizados en el individuo. Se ofrecían exámenes clínicos e incluso atención curativa, reproduciendo la lógica de la atención del sector privado y de su formación académica, que forma e informa al dentista sobre su práctica clínica ${ }^{6,7}$.

En 1952, el Servicio especial de Salud Pública, creado en 1942 como alianza entre el gobierno brasilero y el norteamericano, implementó un programa de atención odontológica en escolares; iniciando así de forma planificada y programada, la primera iniciativa integral de atención odontológica, llamada Sistema Incremental de Atención al Escolar, dirigida a escolares de 6 a 12 años. Al mismo tiempo, se inicia la fluorización del suministro público de agua en Brasil, en Aimorés (Minas Gerais) y Baixo Guandú (Espírito Santo).

En 1957, de forma pionera, el estado de Rio Grande do Sul promulgó una ley (No 3125) en la Asamblea Legislativa, la cual determinó que sea obligatoria la fluorización de las aguas en el estado ${ }^{6,7}$. Y en la década de los '60, con la formación del Instituto Nacional de Seguridad Social (INPS), por la necesidad de unificación de las IAPs se comenzó a acreditar clínicas y consultorios privados odontológicos para brindar asistencia a los trabajadores del mercado formal de trabajo ${ }^{4,6,7}$. Este mantenía la misma lógica de los servicios de salud, basados en acciones curativas y expansión de la red privada de salud ${ }^{8}$.

A lo largo de los años 1970 y 1980, el Ministerio de Salud se ocupaba casi exclusivamente de las campañas nacionales para combatir enfermedades y epidemias. La prestación de servicios de salud a la población estaba bajo responsabilidad del Ministerio de Seguridad Social y su autarquía, que durante dicho periodo cambió de nombre, nuevamente, para INAMPS (Instituto Nacional de Asistencia Médica y Seguridad Social).

Todos estos cambios eran formas administrativas para contener la grave crisis del sector, como resultado de su modelo socialmente injusto, por atender solamente a trabajadores formales en el mercado laboral, y dejando a un enorme contingente de personas a la asistencia de las Santas Casas de Misericordia, beneficencias que desempeñaron un papel de prestación de servicios en salud en la brecha dejada por el poder público; con un presupuesto económicamente insuficiente, pues los recursos de jubilación y pensiones se mezclaban con recursos y gastos en salud; políticamente insostenible, dados los movimientos por una profunda reforma sanitaria en Brasil ${ }^{9-11}$. 
Entre 1964 y 1985, Brasil vivió un período autoritario, en el que el gobierno federal comandado dictatorialmente por militares instauró un modelo de protección social centrado en el pago de servicios privados para la atención de la salud de la población, y basado en la realización de procedimientos, cada vez en aumento. Al mismo tiempo, América Latina en conjunto, vivía un escenario de profunda transformación social y económica, con una transición demográfica y epidemiológica acelerada, con efectos directos sobre el acceso a los servicios de salud y seguridad social, junto con una grave crisis fiscal y deuda estatal ${ }^{11}$. Esto llevó a que el modelo de seguridad social utilizado se derrumbe ${ }^{4}$.

En 1980, se celebró la VII Conferencia Nacional de Salud (CNS), que abarcó conceptos y tendencias de conferencias internacionales de salud, como Alma-Ata, donde la Atención Primaria de Salud (APS) fue colocada como centro del debate sobre políticas públicas del sector y como organizador de toda la estructura de salud. La VII y VIII CNS, esta última celebrada en 1986, constituyeron los pilares conceptuales del futuro sistema universal de salud brasilero (SUS).

A raíz de las Conferencia Nacional de Salud (CNS), los odontólogos salubristas-reformistas también llevaron a cabo, en 1986, la I Conferencia Nacional de Salud Bucal (CNSB) con la misma perspectiva de la VIII CNS: Un cambio radical en el patrón de protección social ofrecido hasta ese momento y la posibilidad de abandonar el modelo del Welfare State tipificado como conservador o corporativo, para adoptar un modelo de seguridad social clasificado como social-demócrata o universal. Esto terminó sucediendo con la promulgación de la Constitución de 1988, que asegura legal y jurídicamente el marco de un nuevo modelo de atención universal de salud, con miras a la descentralización (dando énfasis a la gestión de servicios de salud a nivel local, municipal), atención integral de la salud y participación comunitaria ${ }^{10,12}$.

El objetivo de este documento es evaluar las alteraciones no institucionales en el interior del SUS posteriores a la implementación de la Política Nacional de Salud Bucal.

\section{EI Sistema Único de Salud}

Con base en la seguridad jurídica garantizada por la Constitución de 1988, con respecto a la salud, se crearon dos regulaciones legales que se conocen como leyes orgánicas de salud: la Ley $\mathrm{N}^{\circ} 8.080$, del 19 de septiembre de 1990, y la Ley $\mathrm{N}^{\circ} 8.142$, del 28 diciembre de 1990, las cuales dan forma y contenido para la organización del modelo de salud basado en los principios de acceso universal, descentralización de los servicios a nivel local y atención integral; son los pilares del modelo de protección social conocido como social-demócrata o universal. La primera ley regula los derechos a través de los artículos 196 a 200 de la Constitución Federal de 1988 y la segunda ley establece, entre otros aspectos, la participación popular y la financiación a través del artículo $198^{5,10}$.
Desde la promulgación de la Constitución Federal, queda clara la intención de municipalizar la red de servicios públicos de salud, dado que en el artículo 30, inciso VII, está establecida como competencia municipal "prestar, con la cooperación técnica y financiera de la Unión y del Estado, servicios de atención a la salud de la población". Además, el artículo 7 de la Ley Orgánica de Salud $\mathrm{N}^{\circ} 8.080$, reitera "énfasis en la descentralización de los servicios a los municipios".

El afán municipalista en el Brasil post dictadura fue tan grande que la IX Conferencia Nacional de Salud, realizada en 1992, tuvo como tema central "Salud: la municipalización es el camino". A diferencia de los demás países del mundo que tienen sistemas universales de salud, Brasil se convierte en el único que implanta un sistema de salud público con base municipal. Los demás países diseñaron sus sistemas de salud en bases regionales o distritales con jefaturas sanitarias locales que administraban sus propios presupuestos y organizaban una red de servicios ${ }^{13}$.

Para el Sistema Único de Salud, la lógica detrás de la municipalización de la red de salud era que esta sea ascendente y democrática, localizada lo más cerca posible del usuario y sus necesidades ${ }^{14}$. Sin embargo, este deseo no tomó en cuenta la realidad de los municipios: el 70,5 $\%$ de ellos tiene menos de 20 mil habitantes y el 89,4 $\%$ tiene hasta 50 mil habitantes ${ }^{10,15}$. Los municipios pequeños cuentan con una economía muy frágil (basada principalmente en actividades agropecuarias), que tiene como principal fuente de recaudación los fondos estatales y federales. Esta realidad tributaria del país no favorecía la municipalización de los servicios.

Así un conjunto de servicios y obligaciones con respecto a la atención de la salud se transfería de forma atomizada a los municipios sin tener en cuenta las fuentes de financiamiento para costear estas actividades. En una realidad de más de 5500 municipios existentes, cada uno de ellos pasó a tener la obligación legal de atender a los usuarios en los tres niveles de complejidad del sistema: atención primaria, secundaria (ambulatoria especializada) o terciaria (hospitalaria). La opción de descentralización del sistema tampoco consideró el sistema político electoral establecido en Brasil, basado principalmente en el núcleo municipal. Esto creó interrupciones en la formación de redes distritales/regionales, o consorcios, e incluso puso al sistema de salud en una agenda político-electoral ${ }^{13}$.

Dentro de esta realidad, en la que, por un lado, los municipios no tienen recursos sustanciales para administrar sus responsabilidades de salud, por otro lado el modelo de financiamiento de la salud se produce por inducción a los programas federales de salud. Los municipios y sus redes de salud comienzan a adherirse fuertemente a los más variados programas de salud presentados por el gobierno federal, pues estos presentan "dinero nuevo" en las administraciones locales ${ }^{16}$.

Como la mayoría de los municipios del país dependen en gran medida de fondos federales para cubrir 
sus gastos en salud, las secretarías de salud cuentan con una baja gobernanza en la administración de su red, derivada de una baja autonomía financiera ${ }^{6}$. Además, existe una importante brecha institucional dejada por los estados con respecto al financiamiento de la red de atención a la salud ${ }^{17}$.

En este escenario, el Programa de Salud Familiar (PSF) se implementó en 1994. En los años 1993 y 1996, se establecen dos Normas Operacionales Básicas (NOB): la NOB/SUS 01/93 del 20 de mayo de 1993, y la NOB/ SUS 01/96 del 5 de noviembre de 1996; que establecieron dos nuevas formas de financiamiento: el Piso de Atención Básica (PAB) fijo y variable. Esto hizo que el programa obtuviese un apoyo masivo de los municipios, pues el $\mathrm{PAB}$ variable se introdujo para incentivar la adopción, por parte de los municipios, de programas y estrategias para la organización de Atención Básica a partir de ese modelo. Estos dos marcos legales mencionados pretendían definir las responsabilidades sanitarias de cada esfera gubernamental, con fuerte énfasis en delegar la gestión de la red de servicios de salud a los municipios y fortalecer la transferencia de recursos para financiar acciones de salud ${ }^{18-20}$.

Con una fuente de financiamiento segura y regular que garantizara la ejecución del programa, la adhesión entre los municipios se tornó más fuerte, como se muestra en el Tabla 1. Sin embargo, recién en el año 2000, a través del Decreto Ministerial $N^{\circ} 1.444$, del 28 de diciembre, se determinó la inserción de los equipos de salud bucal en equipos de salud familiar, a través de incentivos financieros para la implementación y costo de las acciones ${ }^{7}$.

\section{Modelo de salud bucal pública en Brasil}

A partir del 2003, la salud bucal se consolidó en el Sistema Único de Salud, con el establecimiento de la Política Nacional de Salud Bucal y el Programa Brasil Sonriente, instaurado por el gobierno federal ${ }^{7}$. A través de un vasto conjunto de marcos legales, el Programa se afianzó como política pública de Estado. Hubo, a partir de entonces, un crecimiento cuantitativo y robusto de los equipos públicos de salud bucal y de odontólogos en el sistema público, lo que consecuentemente permitió un aumento y mayor variedad de procedimientos clínicos odontológicos. El Programa Brasil Sonriente introdujo la salud bucal en el ámbito del SUS y un conjunto de equipos públicos destinados a la creación de una red de cuidados en salud bucal. Como iniciativa de la Política Nacional de Salud Bucal se puede mencionar: un aumento vertiginoso de equipos de salud bucal dentro del Programa Salud de

Tabla 1. Cuantitativo de Equipos de Salud Familiar (eSF) e Equipos de Salud Bucal (eSB)

\begin{tabular}{|c|c|c|c|c|c|c|c|c|}
\hline \multirow{2}{*}{ Año } & \multirow{2}{*}{ Mes } & \multirow{2}{*}{ Población } & \multicolumn{3}{|c|}{ Equipo de Salud Familiar } & \multicolumn{3}{|c|}{ Equipo de Salud Bucal } \\
\hline & & & $\begin{array}{l}\text { № de Municipios } \\
\text { con eSF }\end{array}$ & Instaurados & $\begin{array}{c}\text { Proporción de cobertura } \\
\text { poblacional estimada }\end{array}$ & $\begin{array}{l}\text { № de Municipios } \\
\text { con eSB }\end{array}$ & Instaurados & Instaurados \\
\hline 1998 & 12 & 159644929 & 1134 & 3062 & 6,55 & 0 & 0 & 0 \\
\hline 1999 & 12 & 159644929 & 1646 & 4114 & 8,78 & 0 & 0 & 0 \\
\hline 2000 & 12 & 163957179 & 2762 & 8503 & 17,43 & 0 & 0 & 0 \\
\hline 2001 & 12 & 172385826 & 3682 & 13155 & 25,43 & 1288 & 2002 & 246 \\
\hline 2002 & 12 & 172385826 & 4161 & 16698 & 31,87 & 2302 & 3819 & 442 \\
\hline 2003 & 12 & 174648205 & 4488 & 19068 & 35,69 & 2787 & 5631 & 539 \\
\hline 2004 & 12 & 177243280 & 4664 & 21232 & 38,99 & 3184 & 8234 & 717 \\
\hline 2005 & 12 & 177256093 & 4986 & 24564 & 44,35 & 3897 & 11717 & 886 \\
\hline 2006 & 12 & 185619807 & 5106 & 26729 & 46,19 & 4285 & 14019 & 1067 \\
\hline 2007 & 12 & 188206105 & 5125 & 27324 & 46,62 & 4294 & 14563 & 1131 \\
\hline 2008 & 12 & 188206105 & 5235 & 29300 & 49,51 & 4597 & 16423 & 1384 \\
\hline 2009 & 12 & 189681859 & 5251 & 30328 & 50,69 & 4717 & 17465 & 1517 \\
\hline 2010 & 12 & 191575774 & 5294 & 31660 & 52,23 & 4830 & 18731 & 1693 \\
\hline 2011 & 12 & 190755799 & 5285 & 32295 & 53,41 & 4883 & 19492 & 1933 \\
\hline 2012 & 12 & 192379287 & 5297 & 33404 & 54,84 & 4901 & 20155 & 2048 \\
\hline 2013 & 12 & 193976530 & 5346 & 34715 & 56,37 & 4971 & 21016 & 2134 \\
\hline 2014 & 12 & 193976530 & 5465 & 39310 & 62,48 & 5018 & 22066 & 2257 \\
\hline 2015 & 12 & 193976530 & 5463 & 40162 & 63,72 & 5014 & 22227 & 2240 \\
\hline 2016 & 12 & 193976530 & 5409 & 40098 & 63,70 & 4955 & 22194 & 2190 \\
\hline 2017 & 12 & 206114067 & 5467 & 42119 & 63.91 & 5026 & 232012 & 2689 \\
\hline
\end{tabular}

Fuente: MS/SAS/DAB e IBGE 
la Familia y la creación de los Centros de Especialidades Odontológicas (CEO), de los Laboratorios Regionales de Prótesis Dental (LRPD) y de las Unidades Odontológicas Móviles (UOM), además de acciones intersectoriales en las cuales la salud bucal comienza a participar activamente, por ejemplo, la inclusión de los CEO's en el programa Vivir sin Límites ${ }^{4,18}$ (decreto No 7.612, del 17/11/2011 - Programa constituído con la concepción de crear iniciativas e intensificar acciones en beneficio de las personas con deficiencia) (Figura 1).

A partir del 2005, el Ministerio de Salud pasó a financiar la acreditación de los Laboratorios Regionales de Prótesis Dental. Estos establecimientos pueden ser de naturaleza jurídica pública o privada. Los servicios de prótesis dental que son financiados por el Ministerio de salud son: prótesis totales, parciales removibles y/o coronarias/intraradiculares y fijas/adhesivas. Para acreditar un Laboratorio Regional de Prótesis Dental, no se considera una base poblacional, sino una cantidad de prótesis a ser confeccionadas por mes. Por medio de la Ordenanza Interministerial No 1.646, del 5 de agosto de 2014, se instituye el componente GraduaCeo, como centro de la organización de los servicios de salud bucal. Esta tiene como objetivo integrar las acciones prestadas en los cursos de odontología de las Instituciones de enseñanza superior (IES) a la red municipal de servicios públicos, donde la regulación de vacantes para atención clínica se encuentra bajo la responsabilidad de los gestores públicos de salud, basada en los principios y directrices del SUS ${ }^{21}$.
La manifestación más interesante del Programa Brasil Sonriente ${ }^{7}$ fue la instauración de los Centros de Especialidades Odontológicas (CEO) destinados al soporte matriz de los Equipos de Salud Bucal (eSB) de la Estrategia de Salud de la Familia (ESF). Los CEO son servicios de atención secundaria, constituidos por equipos públicos de referencia de la Atención Primaria para la atención de casos más complejos de asistencia a la salud bucal, integrados al proceso de planeación local-regional ${ }^{22,23}$. Los CEO tienen como función principal ofertar un conjunto de servicios especializados para los equipos de salud bucal de la atención básica, rompiendo con la lógica de oferta de servicios de baja tecnología a la población. Una vez implantado dicho equipo de salud pública, el Ministerio de Salud monitoriza esencialmente las metas mensuales mínimas de un conjunto de procedimientos registrados en el sistema de información ambulatoria - SIA-SUS ${ }^{24}$, para que los municipios puedan recibir un adecuado incentivo financiero federal para costeo (Tabla 2).

Travassos et al. (2006), observaron en un estudio sobre el sistema de salud brasilero como un todo, durante el período de 1998 a 2003, que, por más que se ampliara el acceso, el componente de mayor impacto sobre la frecuencia de utilización de los servicios de salud es la renta, que a su vez está influenciada por la escolaridad. Según este estudio, por más que se amplíe la cobertura por la implementación de nuevos equipos públicos, continúa existiendo desigualdad en lo que se refiere a la utilización de los servicios ${ }^{20}$. Así, al menos en teoría, se

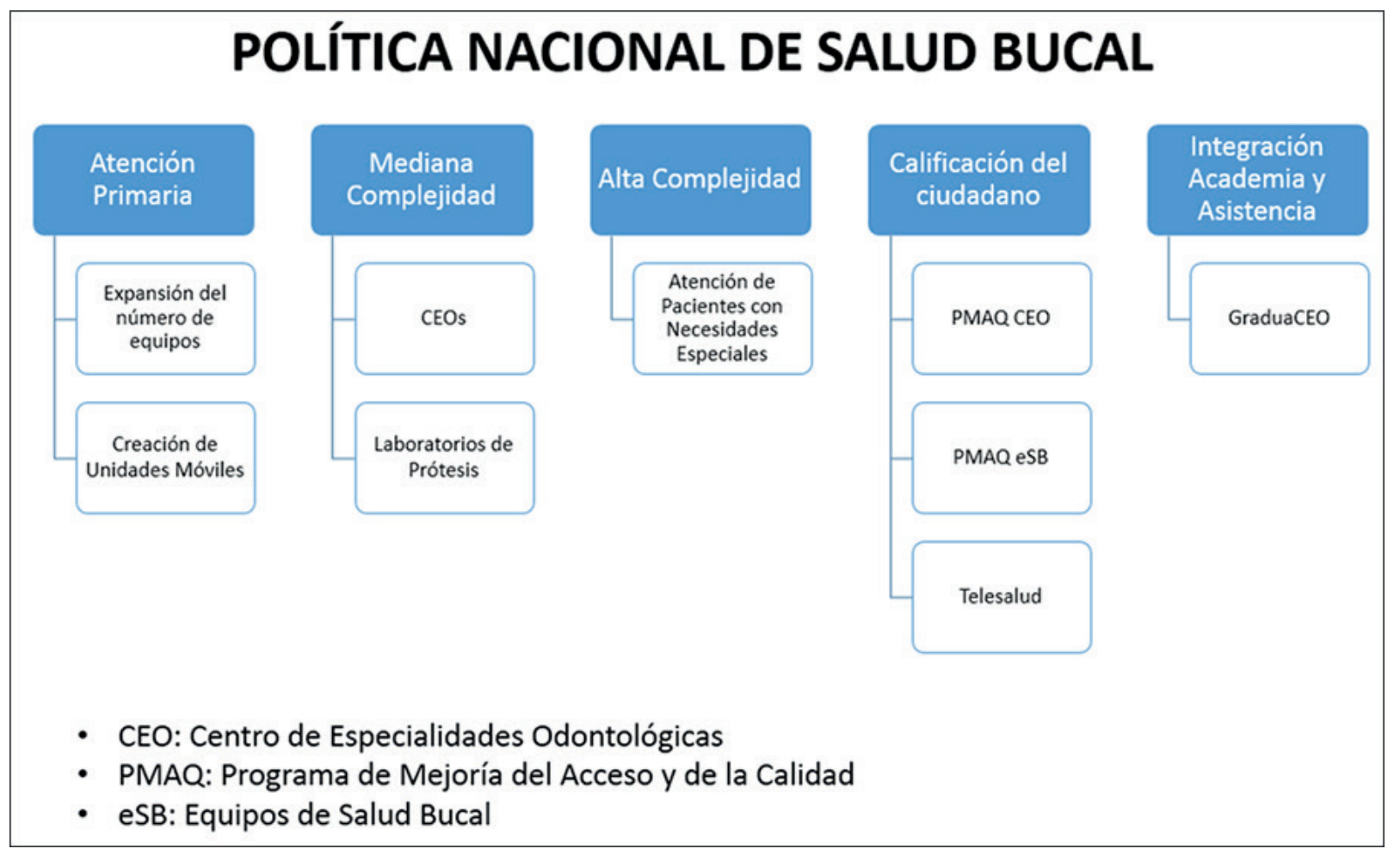

Figura 1. Acciones desarrolladas por la Política Nacional de Salud Bucal Fuente: autor (2020) 
Tabla 2. Acompañamiento de la implementación de la Política Nacional de Salud Bucal

\begin{tabular}{ccccccccc}
\hline Año & $\begin{array}{c}\text { Valor Transferido } \\
\text { del Ministerio } \\
\text { para Municipios } \\
\text { (R\$) }\end{array}$ & $\begin{array}{c}\text { Número de } \\
\text { eSB } \\
\text { Instauradas }\end{array}$ & $\begin{array}{c}\text { \% Cobertura } \\
\text { Pop. }\end{array}$ & $\begin{array}{c}\text { Cantidad de } \\
\text { municipios } \\
\text { con eSB } \\
\text { Instauradas }\end{array}$ & $\begin{array}{c}\text { Valores (R\$) } \\
\text { costeo de los } \\
\text { CEO }\end{array}$ & $\begin{array}{c}\text { Número de } \\
\text { CEO } \\
\text { Instaurados }\end{array}$ & $\begin{array}{c}\text { Número de } \\
\text { Laboratorios }\end{array}$ & $\begin{array}{c}\text { Número de } \\
\text { Prótesis } \\
\text { realizadas }\end{array}$ \\
\hline 2002 & 56885788 & 4261 & $15 \%$ & 2302 & - & - & - & - \\
2003 & 92170448 & 6170 & $20 \%$ & 2787 & - & - & - & - \\
2004 & 197583550 & 8951 & $27 \%$ & 3184 & 4687200 & 100 & - & - \\
2005 & 289374400 & 12602 & $35 \%$ & 3896 & 35811000 & 336 & - & - \\
2006 & 370642800 & 15086 & $40 \%$ & 4285 & 53159600 & 498 & - & - \\
2007 & 407466100 & 15694 & $41 \%$ & 4294 & 63884400 & 604 & - & - \\
2008 & 465559650 & 17801 & $39 \%$ & 4596 & 71498800 & 674 & - & - \\
2009 & 554952700 & 18982 & $48 \%$ & 4717 & 77831550,91 & 808 & - & - \\
2010 & 597217800 & 20424 & $34 \%$ & 4830 & 94346800 & 853 & 676 & 183152 \\
2011 & 651134100 & 21425 & $36 \%$ & 4883 & 93940400 & 882 & 808 & 300978 \\
2012 & 722333880 & 22203 & $37 \%$ & 4901 & 116467250 & 944 & 1351 & 408107 \\
2013 & 748851005 & 23150 & $38 \%$ & 4971 & 159532000 & 988 & 1465 & 462003 \\
2014 & 716528609 & 24243 & $38 \%$ & 5013 & 170620600 & 1032 & 1955 & 600196 \\
2015 & 798935935 & 24467 & 37,82 & 5014 & 196215450 & 1034 & 1770 & 569967 \\
2016 & 80739346 & 24383 & $37 \%$ & 4954 & 200422230 & 1033 & - & - \\
2017 & Indisponível & 25890 & $39 \%$ & 5026 & No disponible & No disponible & No disponible & No disponible \\
\hline
\end{tabular}

Fuente: MS/SAS/DAB e IBGE.

puede afirmar que, por más que se amplíen los equipos públicos de salud, una significativa parcela de usuarios puede continuar sin accesar a los servicios, aun siendo universales y gratuitos. De tal modo, no llegan a mejorar un conjunto de indicadores de salud cuando se analizan poblaciones.

Si en teoría se puede hacer esa suposición, en la práctica, el primer paso para la obtención de acceso a los servicios (demanda) es la existencia física de determinada unidad de salud (oferta), condición primaria para la existencia de una Red. A lo largo de su implementación, la Política Nacional de Salud Bucal (PNSB), bajo el nombre de Brasil Sonriente, proporcionó una vertiginosa ampliación de recursos para la Coordinación Nacional de Salud Bucal, responsable en el interior del Ministerio de Salud por fomentar políticas específicas para salud bucal, aumentando en más de 10 veces en 10 años, en valores absolutos, los recursos disponibles para la creación de una Red de Atención.

Concomitantemente, se amplió el número de equipos de salud bucal implantados en la atención básica, en torno de 3300 equipos en 2002 para más de 24000 equipos en 2016, con más de 1000 centros de especialidades en 2017 ofertando servicios de mediana complejidad odontológica, siendo que no existía ninguno antes del 2003. Se aperturaron también, centenas de Laboratorios Regionales de Prótesis Dental acreditados por el Ministerio de Salud, que abrieron una nueva demanda por oferta de servicios. En este escenario, casi la totalidad de los municipios de Brasil pasó a recibir valores crecientes de atención básica y de mediana complejidad, por contrapartidas federales para inducción de crecimiento de la oferta de asistencia en salud bucal. Es así que, la utilización de servicios de salud no es apenas un reflejo de la decisión individual del usuario, sino un reflejo de los factores externos que determinan su comportamiento y elecciones.

\section{Avances y desafíos}

La Política Nacional de Salud Bucal (PNSB), parte integrante e inherente del Sistema único de Salud, es reconocida por los autores como responsable por el aumento del acceso a los servicios de salud pública; es decir, el programa Brasil Sonriente llevó salud pública a más brasileros y brasileras. Cerca de 15 años después de la implementación de la PNSB, se pueden observar importantes avances en la ampliación de una red de cuidados en salud bucal y consecuente aumento de procedimientos realizados y recursos humanos contratados para efectivización de la política ${ }^{20}$. Se dio un aumento exponencial de cobertura de equipos de salud bucal en la atención básica y una oferta efectiva de servicios de mediana complejidad en salud bucal. Es importante valorizar estos avances significativos. La municipalización del sistema de salud se dio bajo el contexto de redemocratización y con el empoderamiento de los actores locales en la discusión del modelo de salud más conveniente para ofrecer servicios a los usuarios. No obstante, la forma como el Estado brasilero se organiza y se financia deformó la estructura de redes integrales de cuidados en salud.

Algunos estudios ${ }^{6,25-27}$ indicaron que a mayor cobertura, mayor acceso, no siempre resulta en una mayor resolución de una red de atención a la salud bucal, exigiendo 
que la supervisión y evaluación de indicadores sean pieza central en el planeamiento en salud ${ }^{28}$. Un estudio ${ }^{29}$, entre los años 2003 y 2006, evidenciaron el vacío existente con respecto al monitoreo permanente y constante de la expansión mediante indicadores de salud bucal; corroborando con otros estudios ${ }^{6,30}$ que señalan la fragilidad de la evaluación de la calidad de salud por apenas un indicador, por ejemplo, acceso. Otro estudio ${ }^{20}$ afirma que, aunque la oferta de servicios de salud bucal y la utilización de los mismos hayan aumentado, aún existen enormes abismos referentes a las diferencias entre los grupos sociales en la utilización de los servicios, con clara desventaja en los grupos económicamente más frágiles.

Diversos países que poseen sistemas universales de salud cuentan con un modelo de metas de indicadores de salud y de producción a ser alcanzados ${ }^{31}$. Dentro de la Política Nacional de Salud Bucal, se ha evidenciado, por los reportes publicados, un bajo porcentaje de cumplimiento de metas ambulatorias presentadas por los Centros de Especialidades Odontológicas y un débil sistema de regulación, en el cual la mediana complejidad muchas veces sirve como puerta de entrada al sistema, lo que torna urgente la revisión de los parámetros y marcos legales de implementación y funcionamiento.

En la atención primaria, bajo un modelo de salud basado en el territorio y con responsabilidad sanitaria de los equipos sobre determinada población, la elaboración de metas de indicadores de salud bucal orientan la efectividad de servicios ofertados y de la propia Red de Salud Bucal, tanto en la atención básica como en la mediana complejidad odontológica. A través de indicadores de salud bucal, es posible evaluar el alcance de resultados que hayan sido planeados y medir la efectividad de las acciones en salud. Sin embargo, la expansión de los servicios de salud bucal en su orden jurídico no fue regulada por esos indicadores. El alcance de la equidad en el sistema se realiza mediante la medida de indicadores, tornándose nuclear la constante supervisión de la producción ambulatoria y de un conjunto de indicadores de salud bucal para resolver inequidades de acceso a los servicios asistenciales de salud y para la correcta planeación de una Red.

Dicho esto, la efectiva implementación de equipos públicos de salud bucal en el SUS amplió el acceso de los usuarios, lo cual es un importante y admirable avance, pero no creó una Red de salud, que es un concepto mucho más profundo que el simple aumento del número de procedimientos odontológicos realizados: el establecimiento espacial de los Centros de Especialidades Odontológicas (CEO) se dio sin obedecer los criterios de elegibilidad; los protocolos de acceso a los servicios de salud bucal no existen y sistemas de referencia/contra referencia quedan a criterio de los municipios sin que exista una estandarización efectiva de los flujos; indicadores de salud bucal no son observados en la atención básica; la gran mayoría de CEO's no cumplen las metas mínimas de producción y no cuentan con cualquier supervisión que busque mejorarlo; no existe una memoria de cálculo que justifique el porqué de las metas a ser realizadas por los CEO's, fueron establecidas en sus marcos legales específicos; $y$ finalmente, con respecto a la regulación, herramienta fundamental de promoción de equidad, la iniciativa de implantar un sistema de regulación queda a criterio de las prefecturas.

Por más que la implementación de la Política Nacional de Salud Bucal posibilitó un gran salto cuantitativo en el acceso a los servicios públicos de salud bucal en el Sistema único de Salud y se hayan realizado grandes esfuerzos por los gobiernos con respecto a la salud bucal, urge la necesidad de intervenciones más allá del alcance de ella, como con cualquier otra área del sector salud. Cambios y mejoras en los índices epidemiológicos dialogan fuertemente con políticas que superan la asistencia en salud: como inversión en alimentación, vivienda, saneamiento básico, medio ambiente, trabajo, renta, educación, transporte, ocio y acceso a bienes y servicios esenciales. Peres et al. ${ }^{20}$ afirman que el grado de utilización de los servicios de salud bucal sufre influencia directa de dos variables: renta y escolaridad, siendo esas variables intimamente ligadas; cuanto mayor es la escolaridad, mayor también la renta, según análisis poblacionales. El fortalecimiento de una salud pública integral, universal y de calidad que mejore indicadores de salud y normas sanitarias requiere de políticas económicas y sociales, no relativizando la importancia de una correcta y racional política de planeación de asistencia en salud.

\section{Referencias bibliográficas}

1. Mendes EV. As redes de atenção à saúde. 2da ed. Brasília: Organização Pan-Americana da Saúde; 2011.

2. Mercadante OA. Evolução das políticas e do Sistema de saúde no Brasil. En: Finkelman J (Org). Caminhos da saúde pública no Brasil. Rio de Janeiro: Editora Fiocruz, 2002. p. 237-312.

3. Draibe SM. Avaliação de implementação: esboço de uma metodologia de trabalho em políticas públicas. En: Barreira MCRN, Carvalho MCB. (Orgs.). Tendências e perspectivas na avaliaçáo de políticas e programas sociais. São Paulo: IEE/PUC-SP, 2001. p. 13-42.

4. Costa Júnior S. Programa Saúde da Família - Cuidados com o Câncer Bucal: a experiência de Resende, no Estado do Rio de Janeiro. São Paulo: Editora Napoleão 2012.

5. Rodrigues PHA. Desafios políticos para a consolidação do Sistema Único de Saúde: uma abordagem histórica. Hist cienc saúde-Manguinhos. 2014;21(1):37-60. DOI: $10.1590 /$ S0104-59702014000100003.

6. Mattos GCM, Ferreira EF, Leite ICG, Greco RM. A inclusão da equipe de saúde bucal na Estratégia Saúde da Família: entraves, avanços e desafios. Ciênc saúde coletiva. 2014;19(2):373-82. DOI: 10.1590/141381232014192.21652012 .

7. Narvai PC. Avanços e desafios da Política Nacional de Saúde Bucal no Brasil. Rev Tempus Actas de Saúde Coletiva. 2011;5(3):21-34.

8. Fleury S. Reforma sanitária brasileira: dilemas entre o instituinte e o instituído. Ciênc saúde coletiva. 2009;14(3):74352. DOI: 10.1590/S1413-81232009000300010. 
9. Rodrigues PH, Kornis GEM. Os (des)caminhos do direito à saúde no Brasil. Rio de Janeiro; Universidade do Estado do Rio de Janeiro. 1999.

10. Rodrigues PH, Santos IS. Saúde e cidadania: uma visão histórica e comparada do SUS. 2da ed. Rio de Janeiro: Atheneu; 2011.

11. Soares CLM, Paim JS. Aspectos críticos para a implementaçáo da política de saúde bucal no município de Salvador Bahía, Brasil. Cad Saúde Publica 2011;27(5):966-74.

12. Esping-Andersen G. The three worlds of welfare capitalism. New Jersey: Princeton University Press. 1990.

13. Rodrigues PHA. Desafios políticos para a consolidação do Sistema Único de Saúde: uma abordagem histórica. Hist Ciênc Saúde-Manguinhos. 2014;201(1):37-60. DOI: $10.1590 /$ S0104-59702014000100003.

14. Oliveira JLC, Saliba NA. Atenção odontológica no Programa de Saúde da Família de Campos dos Goytacazes. Ciênc Saúde Coletiva. 2005;10(suppl.):297-302. DOI: 10.1590/S1413-81232005000500030.

15. Santos L, Andrade LOM. Redes interfederativas de saúde. En: Silva SF (org). Redes de atenção à saúde no SUS: o pacto pela saúde e redes regionalizadas de açóes e serviços de saúde. Campinas: Idisa - Conasems, 2008. p. 35- 65.

16. Merhy EE. O arcabouço institucional institui, mas também exclui. [2003]. Disponible en: http://www.scielo.br/ $\mathrm{pdf} / \mathrm{csc} / \mathrm{v} 8 \mathrm{n} 2 / \mathrm{a} 03 \mathrm{v} 08 \mathrm{n} 2$. Acceso en: 06/02/2016.

17. Aguilera SLVU, Franca BHS, Moyses ST, Moyses SJ. Articulação entre os níveis de atençáo dos serviços de saúde na Regiáo Metropolitana de Curitiba: desafios para os gestores. Rev Adm Pública. 2013;47(4):1021-40. DOI: 10.1590/S0034-76122013000400010.

18. Pucca Júnior GA, Lucena EHG, Cawahisa PT. Financing national policy on oral health in Brazil in the context of the Unified Health System. Braz Oral Res. 2010;24(Suppl. 1):26-32. DOI: 10.1590/S180683242010000500005 .

19. Piola SF, Servo LMS, de Sá EB, Paiva AB. Estruturas de financiamento e gasto do sistema público de saúde. FUNDAÇÃO OSWALDO CRUZ. A saúde no Brasil em 2030 - prospecção estratégica do sistema de saúde brasileiro: estrutura do financiamento e do gasto setorial [online]. Rio de Janeiro: Fiocruz/Ipea/Ministério da Saúde/Secretaria de Assuntos Estratégicos da Presidência da República; 2013. p. 19-70.

20. Peres KG. Peres MA, Boing AF, Bertoldi AD, Bastos JL, Barros AJD. Reduçáo das desigualdades sociais na utilização de serviços odontológicos no Brasil entre 1998 e 2008. Rev Saúde Pública. 2012;46(2):250-8. DOI: $10.1590 /$ S0034-89102012000200007.

21. BRASIL Portaria Interministerial No 1.646 , de 5 de agosto de 2014. Institui o componente GraduaCEO - Brasil Sorridente, no âmbito da Política Nacional de Saúde Bucal, que irá compor a Rede de Atenção à Saúde (RAS), e dá outras providências. Disponible: http://bvsms.saude. gov.br/bvs/saudelegis/gm/2014/pri1646_05_08_2014. html. Acceso en: 19 de janeiro de 2015.
22. BRASIL Portaria n 599/GM, de 23 de março de 2006.

23. BRASIL Ministério da Saúde. Portaria/MS 648 de 28 de Março de 2006. Aprova a Política Nacional de Atenção Básica, estabelecendo a revisão de diretrizes e normas para a organização da Atenção Básica para o Programa Saúde da Família (PSF) e o Programa Agentes Comunitários de Saúde (PACS). Diário Oficial da República Federativa do Brasil, Brasília, 29 mar. de 2006.

24. Frichembruder K. Centros de Especialidades Odontológicas: Perfil dos coordenadores e dos cirurgióes-dentistas do RS e desenvolvimento de critérios de desempenho. 2011. 131 p. Dissertação (Mestrado em Saúde Bucal Coletiva) - Universidade Federal do Rio Grande do Sul, Porto Alegre, 2011.

25. Rocha RACP, Goes PSA. A Comparação do acesso aos serviços de saúde bucal em áreas cobertas e náo cobertas pela Estratégia de Saúde da Família em Campina Grande, Paraíba, Brasil. Cad Saúde Pública. 2008;24(12):2871-80.

26. Almeida GCM, de Sena MF, Pessoa DMV, Ferreira MAF. Açóes preventivas em saúde bucal desenvolvidas na estratégia de saúde da família: como os dentistas estão avaliando os resultados no seu processo de trabalho? Cad Saúde Coletiva. 2010;18(4):578-86.

27. Pereira CRS. Roncall AG, Cangussu MCT, Noro LRA, Patricio AAR, Lima KC. Impacto da Estratégia de Saúde da Família sobre indicadores de saúde bucal: análise em municípios do Nordeste brasileiro com mais de 100 mil habitantes. Cad Saúde Pública. 2012;28(3):449-62. DOI: 10.1590/S0102-311X2012000300005.

28. Camargo MBJ, Dumith SC, Barros AJD. Uso regular de serviços odontológicos entre adultos: padróes de utilização e tipos de serviços. Cad Saúde Pública. 2009;25(9):1894906. DOI: 10.1590/S0102-311X2009000900004.

29. Souza DS. Visão político-programática da participação da saúde bucal na ESF: uma visão no âmbito estadual. En: Moysés ST, Kriger L, Moysés SJ. Saúde bucal das famílias. São Paulo: Artes Médicas: 2008, p.21-46.

30. Fonseca DAV, Malhe FL, Ambrosano GMB, Pereira AC, Meneghim MC. Influência da organização da atenção básica e das características sociodemográficas da população na demanda pelo pronto atendimento odontológico municipal. Ciênc saúde coletiva. 2012;19(1):269-78. DOI: $10.1590 / 1413-81232014191.2048$.

31. Cordeiro H, Rodrigues PH, Serra CG, Bezerra R. Subsídios para uma proposta de gestão da rede de açóes e serviços de saúde do SUS, com base em resultados. Tempus-Actas de Saúde Coletiva. 2008;2(1):53-62. Apud OHA (ONTARIO HEALTH ASSOCIATON). Regional health Authorities. In Canada, lessons from Ontario. Toronto: OHA; 2002. p. 1 e 16. 